Acta Crystallographica Section D

Biological

Crystallography

ISSN 1399-0047

\section{Karen R. M. Berntsen and Gert Vriend*}

CMBI, Radboud University Medical Center, Geert Grooteplein 26-28, 6525 GA Nijmegen, The Netherlands

Correspondence e-mail: gerrit.vriend@radboudumc.nl

\title{
Anomalies in the refinement of isoleucine
}

A study of isoleucines in protein structures solved using X-ray crystallography revealed a series of systematic trends for the two side-chain torsion angles $\chi_{1}$ and $\chi_{2}$ dependent on the resolution, secondary structure and refinement software used. The average torsion angles for the nine rotamers were similar in high-resolution structures solved using either the REFMAC, CNS or PHENIX software. However, at low resolution these programs often refine towards somewhat different $\chi_{1}$ and $\chi_{2}$ values. Small systematic differences can be observed between refinement software that uses molecular dynamics-type energy terms (for example $C N S$ ) and software that does not use these terms (for example REFMAC). Detailing the standard torsion angles used in refinement software can improve the refinement of protein structures. The target values in the molecular dynamics-type energy functions can also be improved.

\section{Introduction}

In 1991, Engh and Huber published their landmark article on bond lengths and bond angles (Engh \& Huber, 1991). The parameters that they determined are used in nearly all of today's macromolecular software. Other authors have published parameters for planarities (Hooft, Sander et al., 1996b; Sychrovsky et al., 2009; MacArthur \& Thornton, 1996) and torsion angles (Wang et al., 2008; Jones \& Thirup, 1986; Clore \& Kuszewski, 2002; Butterfoss et al., 2005; Hooft et al., 1997; Ponder \& Richards, 1987; Dunbrack \& Cohen, 1997; Lovell et al., 2000), while several groups have been working on the use of torsion angles in refinement software (Clore \& Kuszewski, 2002; Berjanskii et al., 2006; Rice \& Brünger, 1994; Brünger, 1992; Adams et al., 2010). The rotamericity of aminoacid side chains has often been studied for purposes such as homology modelling (Wang et al., 2008), X-ray and NMR refinement (Jones \& Thirup, 1986; Clore \& Kuszewski, 2002), structure validation (Laskowski, MacArthur et al., 1993; Laskowski. Moss et al., 1993; Hooft, Vriend et al., 1996; Lovell et al., 2003; Read et al., 2011) or the determination of MD force-field parameters (Lindorff-Larsen et al., 2010). MacArthur \& Thornton (1999) realised that the average observed values for side-chain torsion angles are resolution-dependent. They concluded that this is caused by the fact that low-occupancy alternate side-chain conformations are often not observable at low resolution, and hypothesized that refinement of a single conformation in density that reflects multiple conformations leads to systematic torsion-angle deviations.

Target values for parameters such as bond lengths and angles are similarly used as data in crystallographic refinement. Consequently, it can also be expected that their final
Received 19 April 2013 Accepted 14 January 2014 
values will be closer to reality at high resolution, when a large amount of X-ray data are available, and closer to the initial target values at low resolution. If these target values can be improved, low-resolution models in particular will improve.

Touw \& Vriend (2010) showed, for example, that the ideal $\tau$ angles depend on secondary structure and amino-acid type, and the values observed in PDB files additionally depend on the resolution and the refinement software used. We studied whether or not these factors similarly influence the observed isoleucine side-chain torsion angles. We conclude that deter-

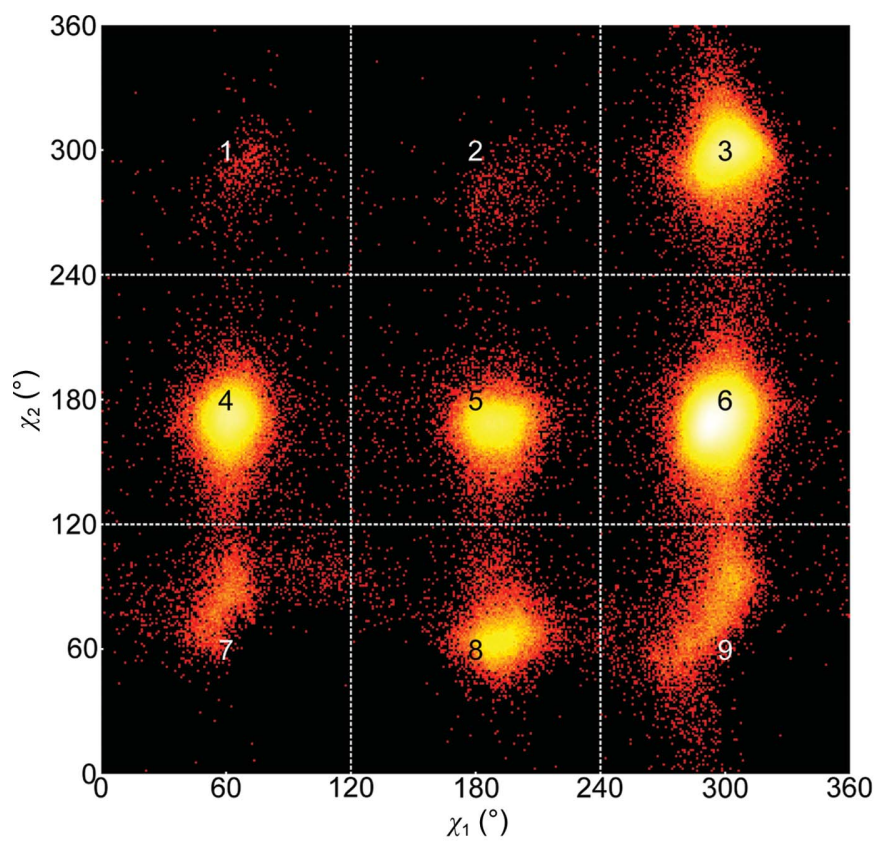

(a)

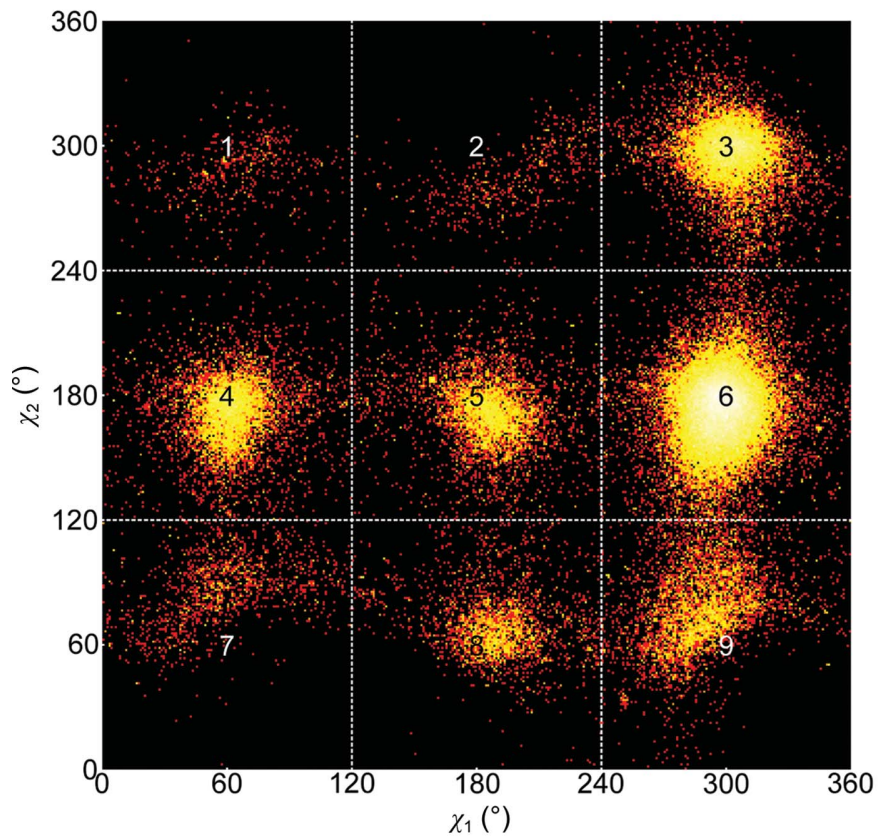

Figure 1

(b) mination of the optimal $\chi_{1}$ and $\chi_{2}$ values is a highly complicated task that the authors of different refinement programs have solved in different ways, all of which are open to improvement. Isoleucine was chosen for a series of reasons: it has two side-chain torsion angles (there are not sufficient data in the PDB to study residues with more variable torsion angles); it is an abundant residue; it is hydrophobic, which means that long-range interactions that are difficult to analyse do not come in play; and it is $\beta$-branched, which means that strong interactions between the side chain and the backbone are involved in rotamer choice.

\section{Methods}

All PDB files that have been solved by X-ray diffraction, that contain at least one intact isoleucine and that were released before February 2013 were extracted from the PDBFinder (Hooft, Sander et al., 1996a) database and stored in a separate relational database (Touw \& Vriend, 2010). A series of scripts were used to extract the data used in this study. The database and all of the software needed to maintain it are available upon request.

Different data-selection protocols were used for different studies. Fig. 6 was based on all isoleucines in the database. Fig. 4 compares today's data on leucine with the data produced in 1999 by MacArthur and Thornton. For this study, we used the leucines in a PDB_SELECT (Hooft, Sander et al., 1996b; http://swift.cmbi.ru.nl/gv/select/) data set of 21667 protein chains that had been solved by X-ray diffraction methods at a resolution better than $3.0 \AA$ and that had an $R$ factor lower than 0.25 . This data set contains no pairs of

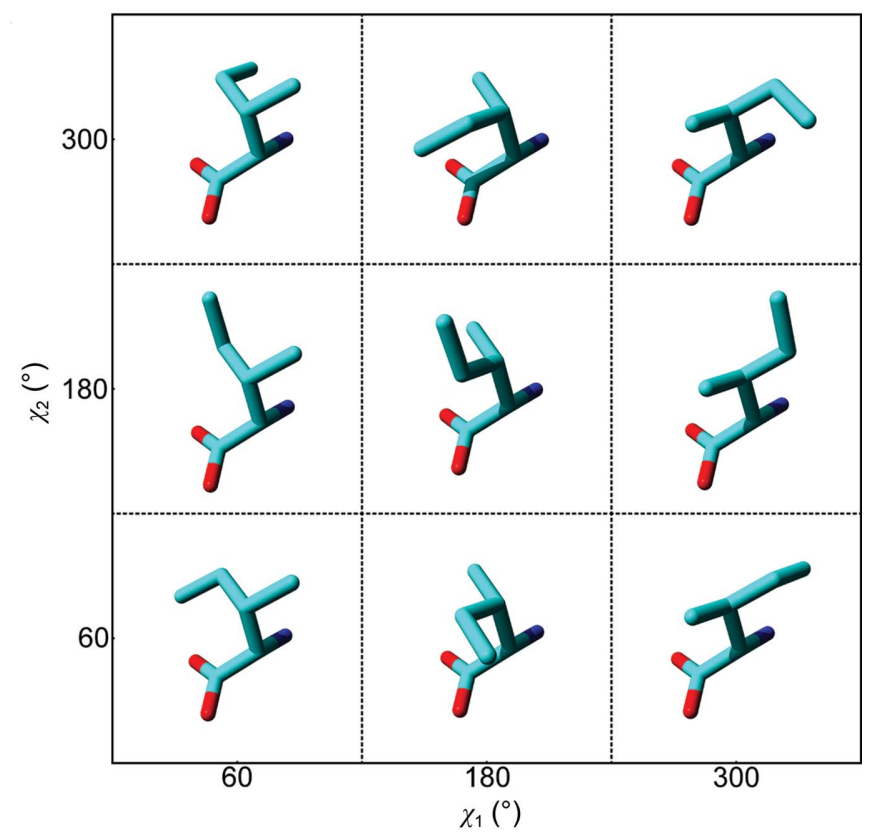

(c)

Two-dimensional histograms of the $\chi_{1}$ and $\chi_{2}$ dihedral angles of isoleucine in structures with resolution ranges of $(a) 0.0-2.0 \AA$ and $(b) 3.0-10.0 \AA$. The data are scaled logarithmically from black (minimum) to white (maximum) and are divided into nine sections of $120 \times 120^{\circ}$. The bin size is $1^{\circ}$. A total of 558287 isoleucines contributed to the high-resolution plot $(a)$ and 90891 contributed to the low-resolution plot $(b)$. $(c)$ The nine rotamers of isoleucine with $\chi$ values of $60.0^{\circ}$ (gauche or $\left.^{+}\right), 180.0^{\circ}$ (trans or $\mathrm{t}$ ) or $300.0^{\circ}\left(\right.$ gauche $^{-}$or $\mathrm{g}^{-}$). 
sequences that are more than $90 \%$ identical upon sequence alignment. Figs. 1, 2, 5, 7(g), 7(h), 8 and 9 are based on the 1765734 isoleucines that fulfilled all of the criteria listed in Table 1.

The database was augmented with a large number of computationally derived parameters, such as torsion angles, secondary structure, $\varphi$ and $\psi$, and residual WHAT_CHECK (Hooft, Vriend et al., 1996) quality parameters (summarized in Table 1). These parameters were obtained using the WHAT IF (Vriend, 1990) web service (Hekkelman et al., 2010) and DSSP (Kabsch \& Sander, 1983; Joosten, te Beek et al., 2011).

Electron densities from EDS (Kleywegt et al., 2004) were inspected using Coot (Emsley \& Cowtan, 2004) and screenshots were obtained using PyMOL (v.1.2.0.1; Schrödinger) and YASARA (Krieger et al., 2002).

Plots were generated using the statistical language $\mathrm{R}$ ( $\mathrm{R}$ Development Core Team, 2012), employing the plyr (Wickham, 2011) package for data aggregation, and twodimensional histograms were generated using Java and GIMP (Kimball et al., 1997).

The YASARA (Krieger et al., 2002) macro for Python (Lutz, 2001) was used to isolate the first $\alpha$-helix (Ser6-Cys16) from crambin (PDB entry 3nir; Schmidt et al., 2011) and to mutate all of its residues to alanines. The hydrogen positions in this $\alpha$-helix were determined using the method of Hooft and coworkers (Hooft, Sander et al., 1996c; Krieger et al., 2012). This $\alpha$-helix was energy-minimized in a simulation cell in vacuum using the NOVA (Krieger et al., 2002) force field, with nonperiodic boundaries, a $20 \AA$ cutoff for nonbonded interactions and YASARA's Simspeed parameter set to 'slow'. A short 'steepest-descent minimization' was followed by simulated annealing (200 steps of $2 \mathrm{fs}$; at every tenth step the atom velocities were reduced by 0.9 ) until the energy improvement was less than $0.05 \mathrm{~kJ} \mathrm{~mol}^{-1}$ per atom. After this minimization, Ala11 was mutated to an isoleucine $\left(\chi_{1}=0, \chi_{2}=0\right)$ which was then rotated around its $\chi_{1}$ and $\chi_{2}$ in steps of $1.0^{\circ}$ to obtain $360^{2}$ different conformations. For each conformation, the total energy (in $\mathrm{J} \mathrm{mol}^{-1}$ ) of the system and the atom experiencing
Table 1

Selection criteria for isoleucines.

\begin{tabular}{|c|c|}
\hline Selection parameter & Criterion \\
\hline Experimental $n$ & X-ray \\
\hline Struct & $\begin{array}{l}\text { Not N-terminal or C-terminal, not next to } \\
\text { a terminus and not next to Gly, Pro or } \\
\text { a noncanonical residue }\end{array}$ \\
\hline Side-chain and backbone atoms & $\begin{array}{l}\text { Not mentioned in the WHAT_CHECK } \\
\text { (Hooft, Vriend et al., 1996) quality } \\
\text { report calculated with the WHAT IF } \\
\text { web service (Hekkelman et al., 2010) } \dagger\end{array}$ \\
\hline$B$ factor, $\mathrm{C}^{\beta}$ and backbone atoms & $\begin{array}{l}<60.0 \AA^{2} \text { or }<2.5 \times \text { the average } B \text { factor } \\
\quad \text { over } \mathrm{C}^{\alpha}\end{array}$ \\
\hline$D S S P$ secondary structure & $\mathrm{H}, \mathrm{E}$ or loop \\
\hline
\end{tabular}

$\dagger$ This extensive list of criteria is briefly explained at http://swift.cmbi.ru.nl/gv/isoleucine/. The criteria include, for example, atomic clashes, spurious covalent bonds, missing atoms etc.

the greatest force were stored and used to colour the $\ln \left(\right.$ energy) contours in the $\chi_{1} / \chi_{2}$ energy plots. Atom distance contour plots were produced in a similar way. For this plot, Ile11 was again rotated into $360^{2}$ different conformations. All methyl and methylene groups were replaced by pseudo-atoms $\left(\mathrm{MB}\right.$ for the $\mathrm{C}^{\beta}$ of alanine and $\mathrm{QG}, \mathrm{MG}$ and $\mathrm{MD}$ for $\mathrm{C}^{\gamma 1}, \mathrm{C}^{\gamma 2}$ and $\mathrm{C}^{\delta 1}$ of isoleucine, respectively) located at their centres of mass. For every conformation, the distances from QG, MG and MD of Ile11 to all other (pseudo) atoms (excluding 1-2 and 13 interactions) were calculated. These distances were corrected by the van der Waals radii of the atoms involved $(\mathrm{H}$,

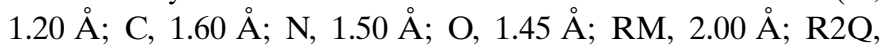
$2.00 \AA$; Tsai, 2007). The minimum resulting distance per conformation was plotted on a contour plot and the corresponding atom pairs were used as labels to colour this plot. The same two procedures were followed for the $\beta$-hairpin Val13-Gln26 from the VP2 subunit of human rhinovirus (PDB entry 1hrv; Rosenwirth et al., 1995), in which Gly19 (in the $\beta$-turn) was not mutated and Ala23 was mutated back to isoleucine.

Two-dimensional histograms of $\chi_{1} / \chi_{2}$ distributions were fitted against a two-dimensional Gaussian function to deter-
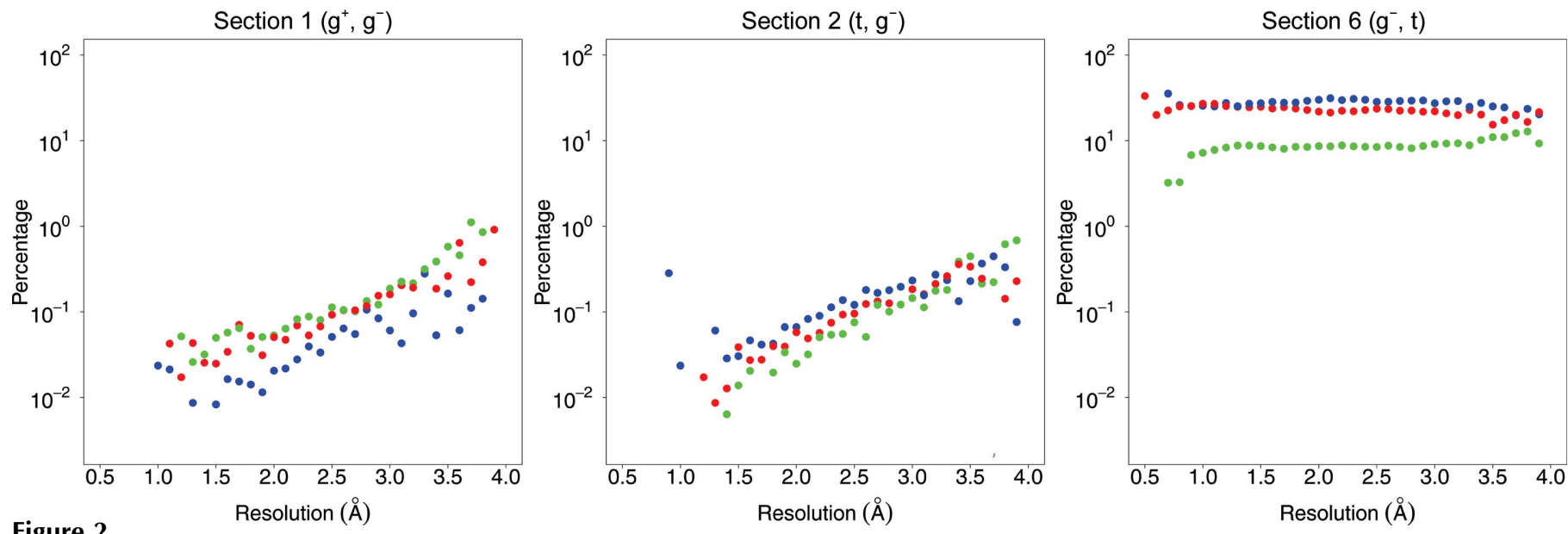

Percentage of isoleucines in three sections as a function of secondary structure and resolution. The sections are as indicated in Fig. 1. Each dot represents the percentage of all isoleucines in a $0.1 \AA$ wide resolution bin that have a $\chi_{1}-\chi_{2}$ combination corresponding to that section, and the secondary structure as indicated by the colour. Only isoleucines that fulfil the quality criteria of Table 1 are represented. $\alpha$-Helix, blue; $\beta$-strand, red; loop, green. 
mine the local maxima and two-dimensional spreads in each of the nine rotamer sections.

\section{Results}

The rotamers of isoleucine can be divided into nine sections, which are highly unevenly populated (see Fig. 1). This is mainly caused by strain owing to interactions between the $\mathrm{C}^{\gamma}$ and $\mathrm{C}^{\delta}$ atoms of the side chain and atoms in the local backbone. The low population of sections 1 and 2 became even more pronounced when we only analysed high-resolution structures (Fig. 1a).

Fig. 2 shows the occurrence of $\chi_{1}-\chi_{2}$ combinations as a function of resolution and secondary structure. At higher
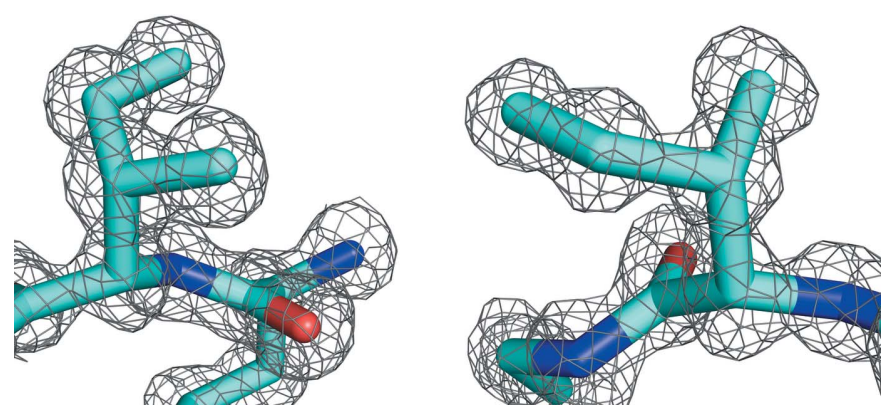

\section{Figure 3}

Two examples of rare isoleucine rotamers that were unambiguously observed in good electron density in structures determined at around $1.0 \AA$ resolution. Left: Ile337 in chain $A$ of the triacylglycerol lipase protein (PDB entry 1d5t; Brzozowski et al., 2000) is observed in section 1. Right: Ile15 in chain $B$ of the HIV protease (PDB entry 1kzk; Reiling et al. 2002) is observed in section 2. Electron densities were obtained from the EDS server and are contoured at $1.4 \sigma$. At http://swift.cmbi.ru.nl/gv/ isoleucine/ the local structures of these residues are shown and the interactions that keep the residues in these unfavourable rotamers are explained. MolProbity (Chen et al., 2010) and WHAT_CHECK (Hooft, Vriend et al., 1996) call these two rotamers 'poor'. We believe that 'improbable' is a better description.

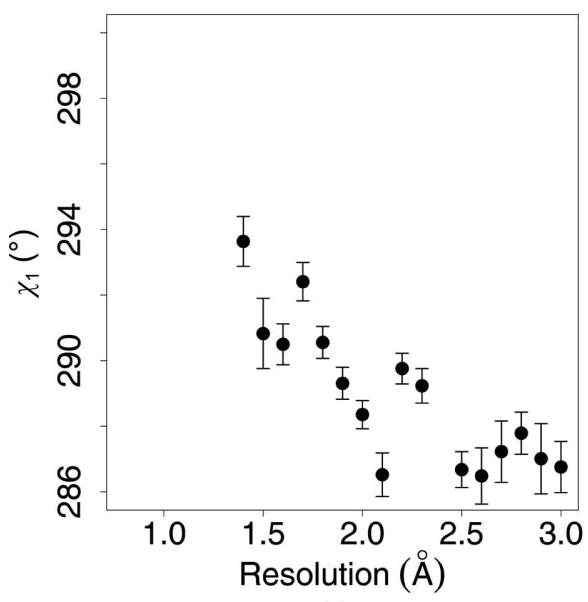

(a)

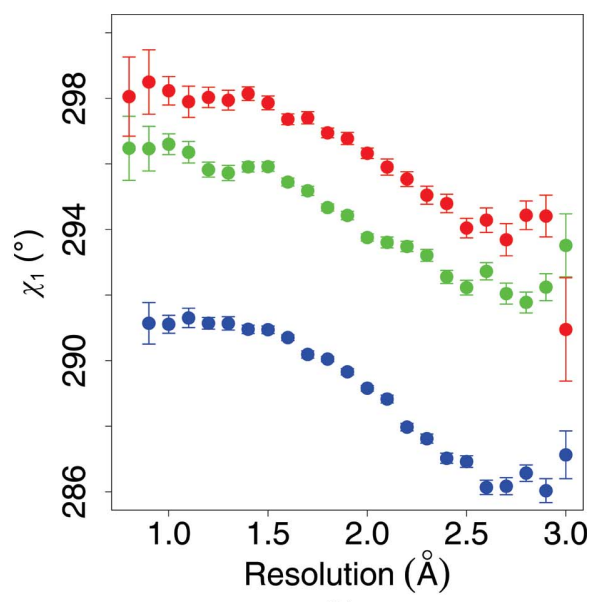

(b)
Figure 4

Mean value of the $\chi_{1}$ gauche $^{-}$rotamer as a function of resolution and secondary structure for leucine (independent of $\chi_{2}$ ). (a) Values reconstructed from the study of MacArthur \& Thornton (1999). (b) As in (a) but for a much larger data set (see \$2) and subdivided for secondary structure. Colour coding is the same as in Fig. 2. Each dot represents at least 50 observations. Error bars represent the standard errors of the mean. resolution, we observe significantly lower populations in sections 1, 2, 7 and 9. Fig. 2 shows the relative frequencies of isoleucines in sections 1,2 and 6 as a function of resolution and secondary structure.

As illustrated in Fig. 2, there are around ten times fewer isoleucines in sections 1 and 2 at high resolution $(\sim 1.5 \AA)$ than at low resolution $(\sim 3.0 \AA)$. The population differences of sections 1 and 2 (see Figs. 1 and 2) suggest that the incidence of isoleucines in these sections could be zero if we look solely at structures solved at extremely high resolution. However, inspection of two examples (see Fig. 3) reveals that $\chi_{1}-\chi_{2}$ torsion-angle combinations corresponding to sections 1 and 2 do occur in high-resolution protein structures. We compared all isoleucines in the original PDB files with the corresponding isoleucines in the PDB_REDO databank (Joosten \& Vriend, 2007; Joosten, Joosten et al., 2011). We studied 951631 isoleucines that have all atomic $B$ factors less than $80 \AA^{2}$ and all atomic occupancies of 1.0 in both databases. We observe that sections 1 and 2 are less populated in the $P D B_{-} R E D O$ files. In most cases we also observe that $\chi_{2}$ is different. A full list of observed differences (also as a function of secondary structure, in absolute and relative terms) is available from http://swift.cmbi.ru.nl/gv/isoleucine/.

In 1999, MacArthur and Thornton studied the resolutiondependence of $\chi_{1}$ for the 17 relevant amino-acid types (MacArthur \& Thornton, 1999). At the time, they could only study $\chi_{1}$ because they did not have sufficient data to study details such as multiple side-chain torsion angles or secondary structure. They did, however, show a detailed plot for the gauche $e^{-}$angles of leucine as a function of resolution. Purely for reference, we compared this plot with the values that we obtained, split up into three secondary-structure types. Fig. 4 shows the resolution-dependence of $\chi_{1}$ in leucine as determined by MacArthur and Thornton in 1999 and by our group in 2013. This plot deals with leucine rather than isoleucine because MacArthur and Thornton presented data for leucine but not for isoleucine. Fig. 4 confirms the previously observed resolutiondependence of the torsion angles, but it also shows that this dependence is more complicated than the near-linear behaviour observed in 1999.

Fig. 5 shows the average isoleucine side-chain torsion angles in the nine sections of Fig. 1 as a function of resolution and secondary structure. This figure shows that these values not only differ between different resolutions and secondary structures, but that the resolution dependence also differs between the nine sections. When comparing a single row or column in Fig. 5, the average value of one $\chi$ often depends on the rotamer of the other $\chi$.

It is tempting to look for trends in the 18 panels of Fig. 5. For example, the mean value of the $\chi_{1}$ gauche ${ }^{-}$rotamer is 
always largest for $\beta$-strand and smallest for $\alpha$-helix, independent of $\chi_{2}$, while the mean value of the $\chi_{1}$ trans rotamer is always smallest in $\beta$-strand. The $\chi_{1}$ gauche $e^{+}$rotamer, however, does not show such trends. The average values in a loop often lie between the average values in $\alpha$-helix and $\beta$-strand, but in five of the 18 panels this is not the case. $\chi_{1}$ is more strongly influenced by the secondary structure than $\chi_{2}$, as might be expected from the smaller distance between $\chi_{1}$ and the backbone compared with the distance between $\chi_{2}$ and the backbone. The secondary structure has the least influence on $\chi_{2}$ when it adopts a trans conformation, corresponding to the $\mathrm{C}^{\delta}$ atom pointing away from the backbone. As expected, the combination $\chi_{1}, \chi_{2}=$ gauche $^{+}$, trans shows the least secondary-structure influence on both $\chi_{1}$ and $\chi_{2}$. However, the
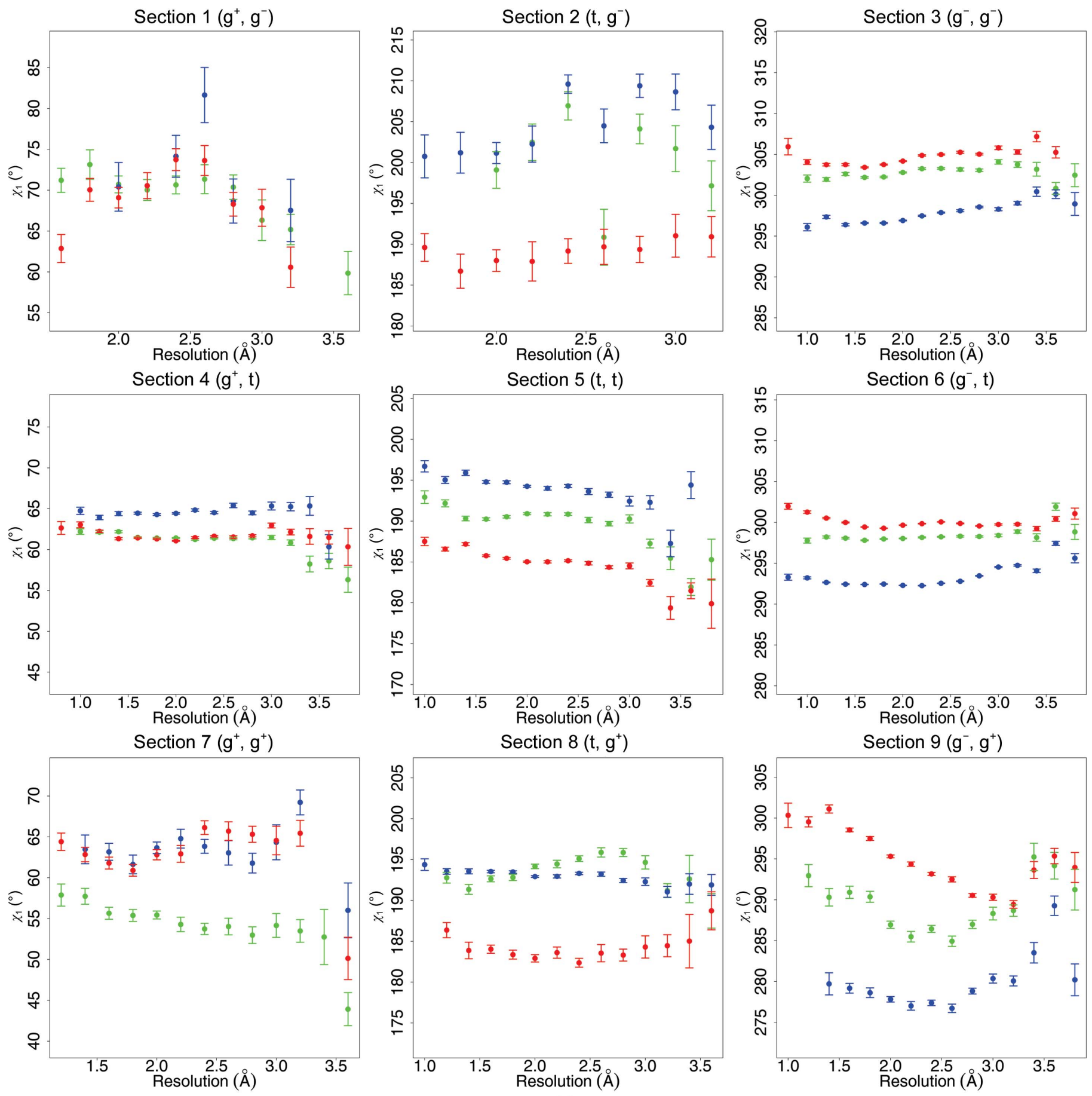

Figure 5

(a)

The average side-chain torsion-angle values of Ile as a function of resolution and secondary structure. Each dot represents the mean of at least 50 data points. Error bars represent the standard errors of the mean. The nine panels in $(a)$ show $\chi_{1}$ values. The nine panels in $(b)$ show $\chi_{2}$ values. Colours are the same as in Fig. 2. In both $(a)$ and $(b)$, the panels are placed in the same order as in Fig. 1. Consequently, similar torsion angles are oriented vertically in $(a)$ and horizontally in $(b)$. The resolution bin size is $0.2 \AA$. 
differences in the mean values are only marginally significant.

A series of studies on the dependence of the backbone angle $\tau$ on resolution, secondary structure and the refinement software used (Touw \& Vriend, 2010; Jaskolski et al., 2007; Karplus, 1996; Chakrabarti \& Pal, 2001; Jiang et al., 2010; Lundgren \& Niemi, 2012) revealed the importance of using the secondary structure as determined by DSSP (Kabsch \& Sander, 1983; Joosten, te Beek et al., 2011), rather than using backbone torsion angles as a description of that secondary structure. The cooperative effects caused by the torsion-angle repeats and the repeat pattern of the hydrogen bonds cause systematic deviations of $\tau$ angles from their most relaxed value. Fig. 6 is a variant of Fig. 5. In Fig. 6 only those residues in a $\alpha$-helix or $\beta$-strand are shown that have $\varphi, \psi$ angles in agreement with the secondary structure (Touw \& Vriend, 2010). The residues observed in loops according to DSSP (green in Figs. 2, 4 and 5) have been split in Fig. 6 into three categories, depending on whether their $\varphi, \psi$ angles correspond to those of an $\alpha$-helix, a $\beta$-strand or neither.
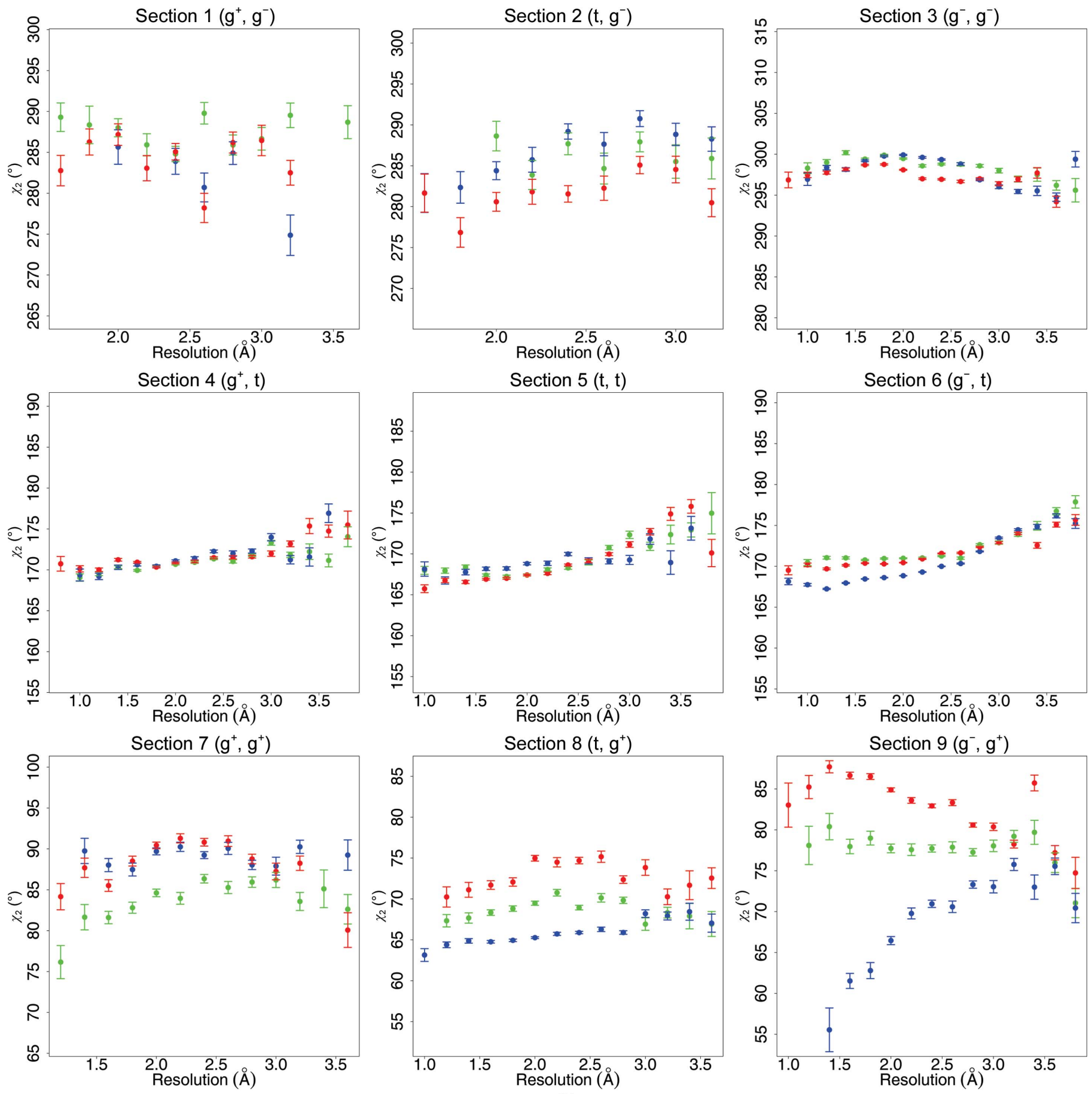

(b)

Figure 5 (continued)

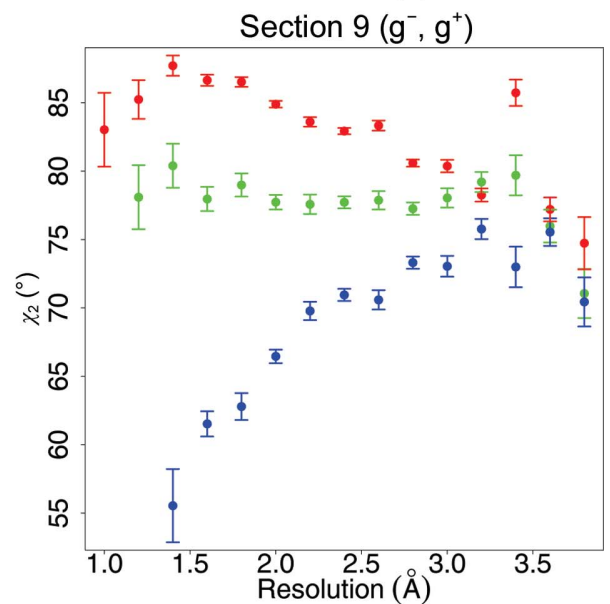


In most panels, the loop residues with $\alpha$-helix and $\beta$-strand $\varphi, \psi$ angles have more similar $\chi_{1}$ and $\chi_{2}$ angles than the residues actually in an $\alpha$-helix and $\beta$-strand according to DSSP. The differences between the $\chi_{1}$ and $\chi_{2}$ angles in the three subcategories of the loop residues nevertheless tend to be significant. In Figs. 5 and 6 , section $9\left(\chi_{1}, \chi_{2}=\right.$ gauche $^{-}$, gauch $^{+}$) shows the most extreme resolution-dependence. This section also stands out in Fig. 1.

The results shown in Figs. 1-6 are the result of a complex interplay between a large number of attractive and repulsive forces between atoms in the side chain and those in the local backbone. The fact that the isoleucines are observed in whole
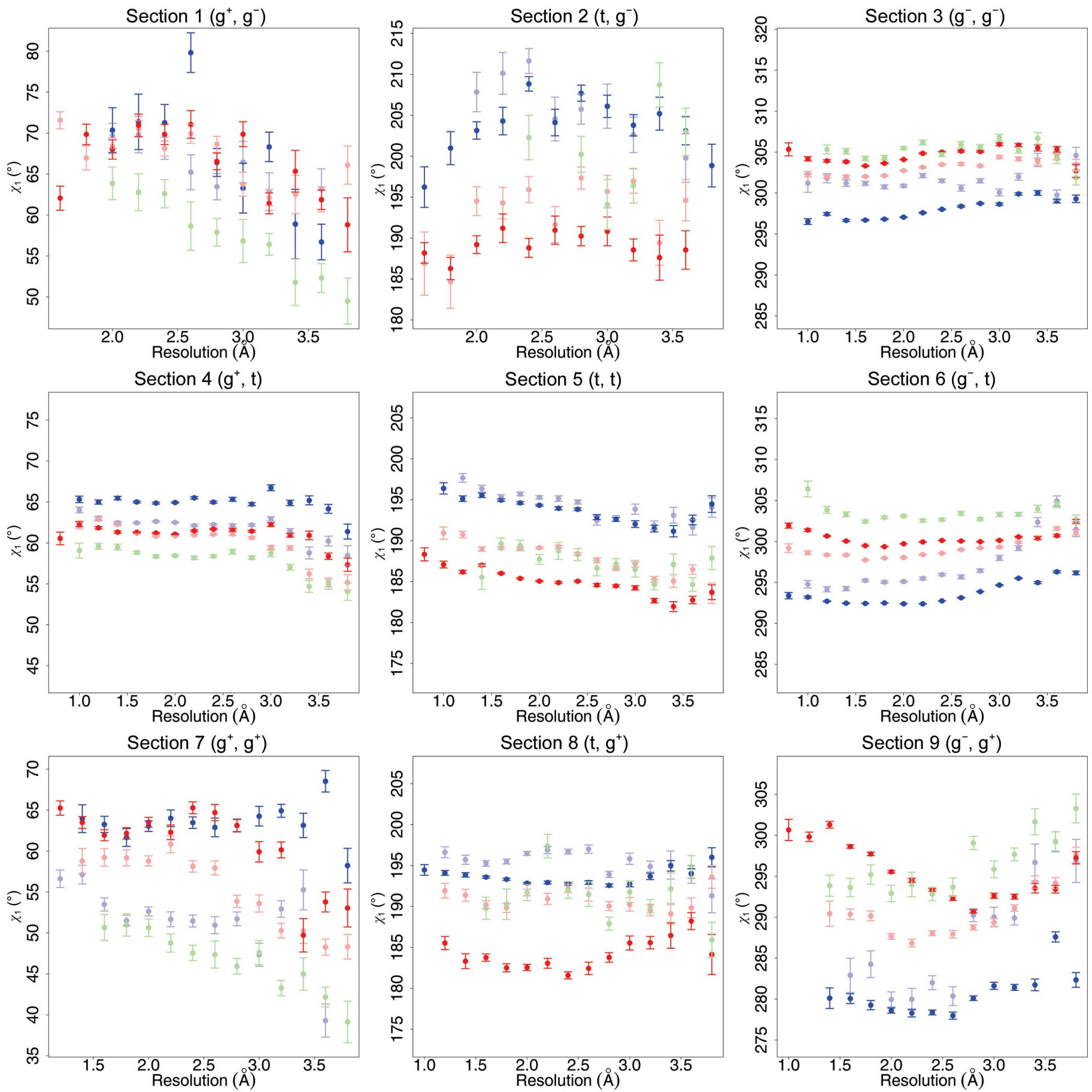

Figure 6

(a)

The average side-chain torsion angles of isoleucine as a function of resolution, secondary structure and backbone dihedrals. Error bars represent the standard errors of the mean. The nine panels in $(a)$ show $\chi_{1}$ values. The nine panels in $(b)$ show $\chi_{2}$ values. In both $(a)$ and $(b)$, the panels are placed in the same order as in Fig. 1. Dark blue and red are for isoleucine in an $\alpha$-helix or $\beta$-strand, respectively, that have the corresponding backbone $\varphi, \psi$ angles. The lighter coloured blue, red and green data are isoleucines that are located in a loop according to DSSP, but that have $\varphi, \psi$ angles as in an $\alpha$-helix, $\beta$-strand or neither, respectively. Isoleucines without missing atoms were taken from virtually every suitable X-ray structure in the PDB to obtain acceptable counting statistics. The resolution bin size was $0.2 \AA$, so that each dot represents the mean of at least 50 data points. 
proteins, in which three-dimensional contacts with other residues occur, means that the plots are inevitably complicated by noise. Figs. 1-6 show that the number of local minima in the conformational space of isoleucine is limited. They also show that these minima are not typically observed with angles of 60 , 180 or $300^{\circ}$, as could be expected from a naive interpretation of $s p^{3}$ hybridization of the atoms involved. Fig. 7 illustrates those forces that can contribute to this observation.

For two typical backbone conformations (as in Figs. $7 a$ and $7 b)$, Figs. $7(c)$ and $7(d)$ show which atom feels the largest overall force when the two side-chain torsion angles are rotated in the entire conformational space in $360 \times 360$ steps of $1^{\circ}$. In general terms, we observed that the conformations that are most populated in experimentally determined protein structures (Fig. $7 g$ and $7 h$ ) correspond to areas where the energy (as computed with YASARA using the YASARA NOVA force field; Krieger et al., 2002) is optimal. Neither protein structures nor force fields are perfect yet, so the computed local minima in $\chi_{1}-\chi_{2}$ space do not correspond perfectly with the observed maxima in the frequency plots.
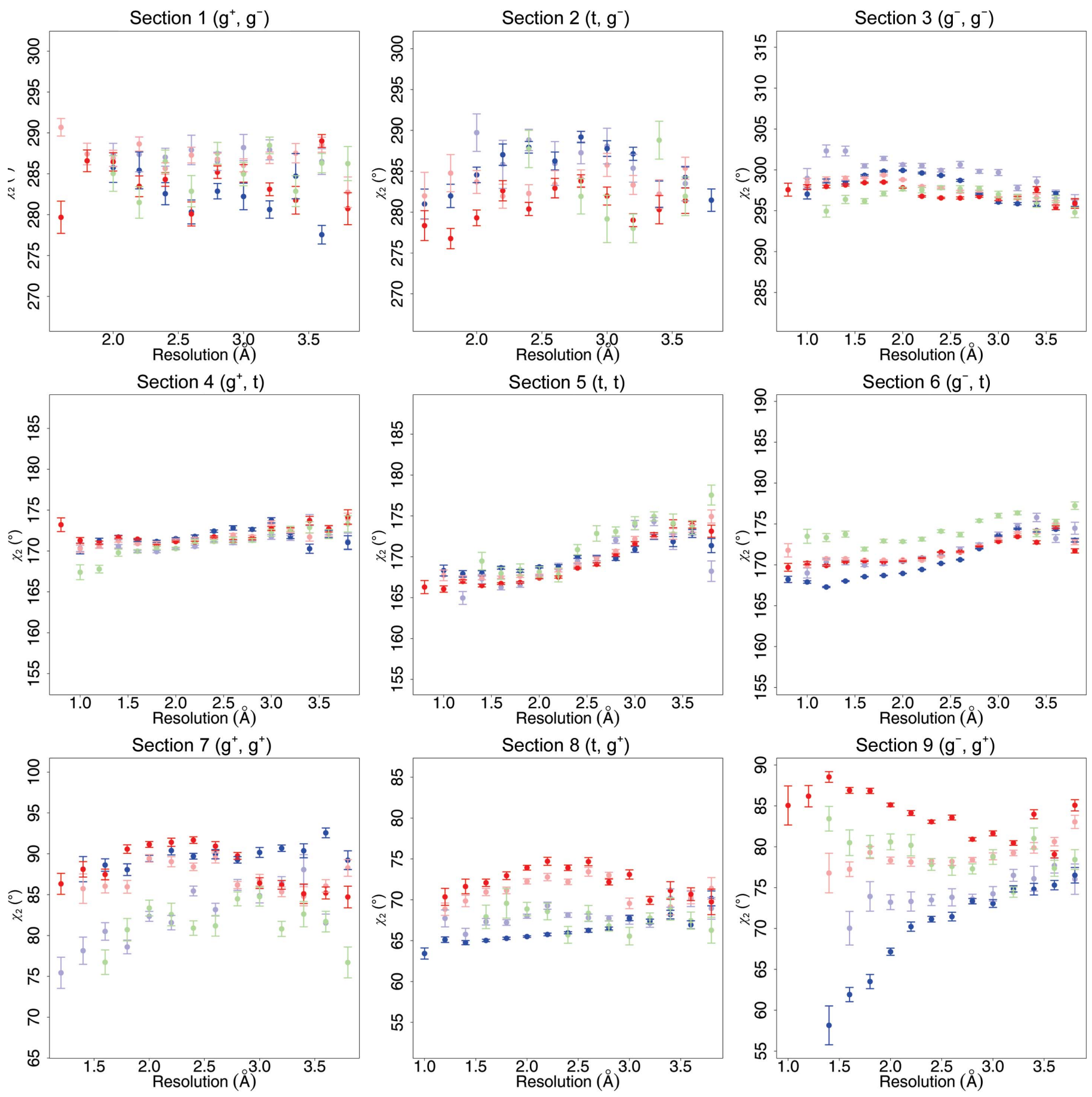

Figure 6 (continued)

(b) 
However, the location and depth of the local minima in the energy plots correspond well enough with the maxima in the frequency plots to explain the observed frequency differences in the $2 \times 9$ sections in Fig. 7. For example, section 4 is relatively less populated in $\alpha$-helix than in $\beta$-strand. This is caused by the close proximity of the isoleucine $\mathrm{C}^{\delta}$ atom to the $\mathrm{H}^{\alpha}$ atom of the alanine one turn away in the $\alpha$-helix. This contact does not exist in the strand situation. Repulsive forces between the isoleucine $\mathrm{C}^{\delta}$ atom and the backbone $\mathrm{O}$ atom of the residue four positions earlier in the $\alpha$-helix cause section 9 in the $\alpha$-helix to be little populated. Section 9 in the $\beta$-strand, on the other hand, is intermediately populated. The difference in population of section 8 is fully explained by repulsive forces observed in the strand situation (with its own backbone $\mathrm{H}^{\alpha}$ atom and the $\mathrm{N}$ and $\mathrm{N}-\mathrm{H}$ of the next residue) that are absent in the helix. The top half of the banana-shaped population distribution in section 9 falls in an area that is a local energy minimum in the YASARA NOVA force field. The bottom half of this banana shape is situated in an area that YASARA NOVA considers less favourable because of repulsive interactions between the $\mathrm{C}^{\delta}$ atom and the $\mathrm{C}, \mathrm{O}$ and $\mathrm{N}$ atoms of the peptide plane just before the isoleucine.

Fig. 8 shows that the observed local optima in isoleucine's $\chi_{1}-\chi_{2}$ space do not agree with the optima derived from the YASARA NOVA (Krieger et al., 2002) force field in all sections. Obviously, this disagreement does not have any

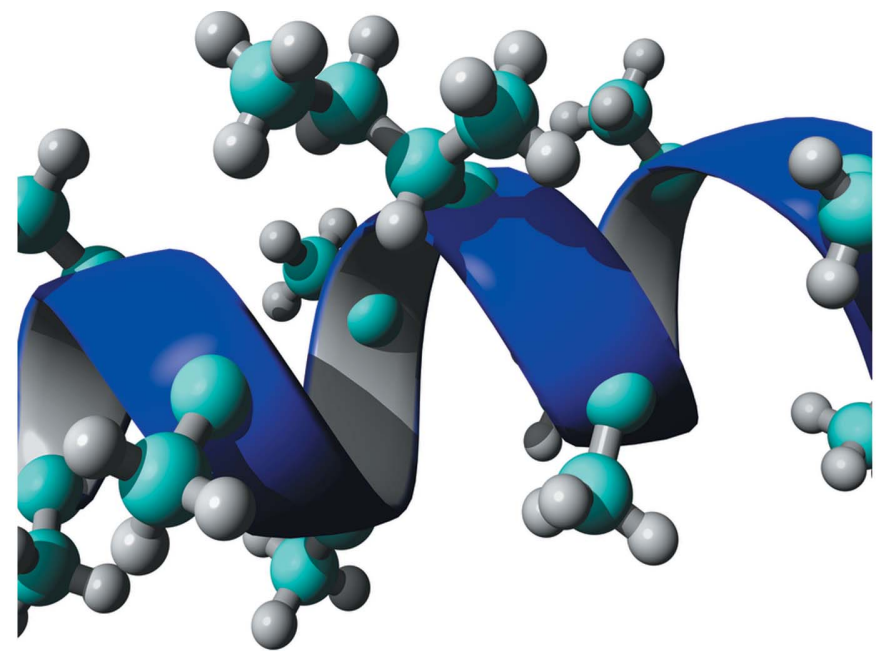

(a)

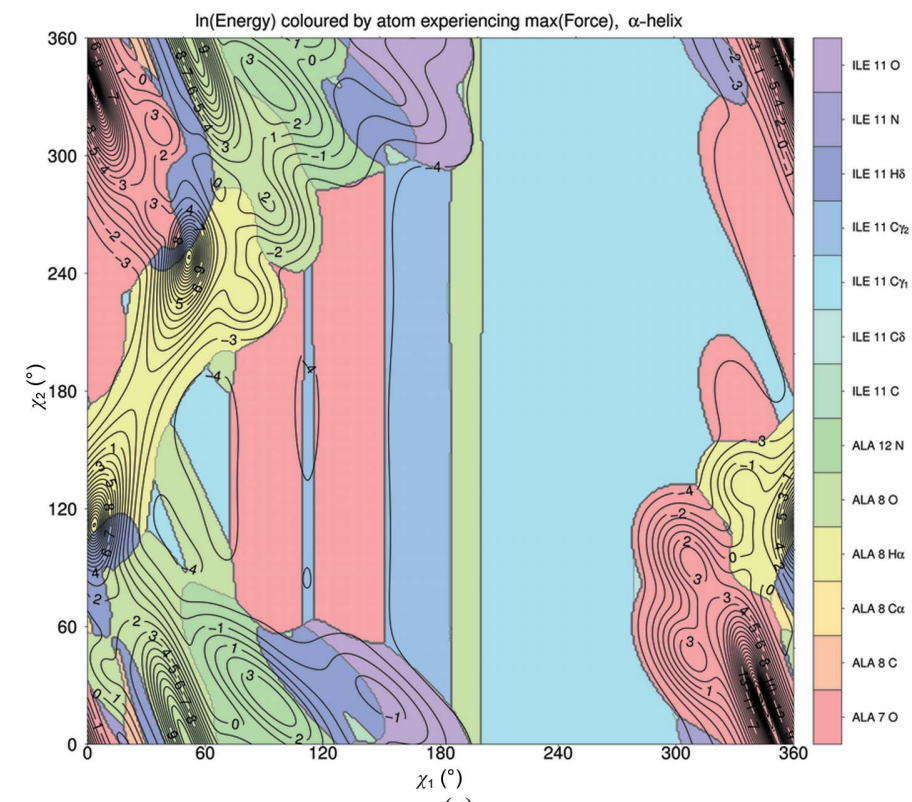

(c)

Figure 7

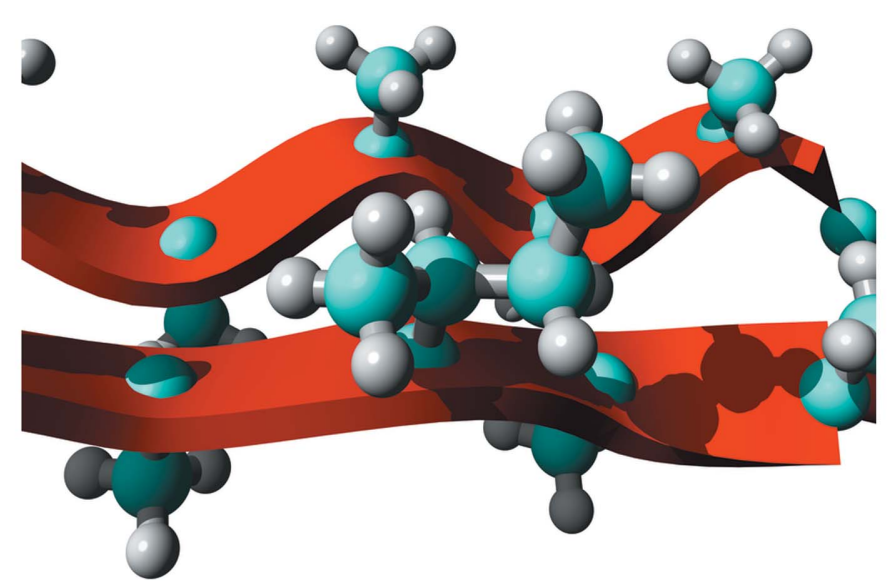

(b)

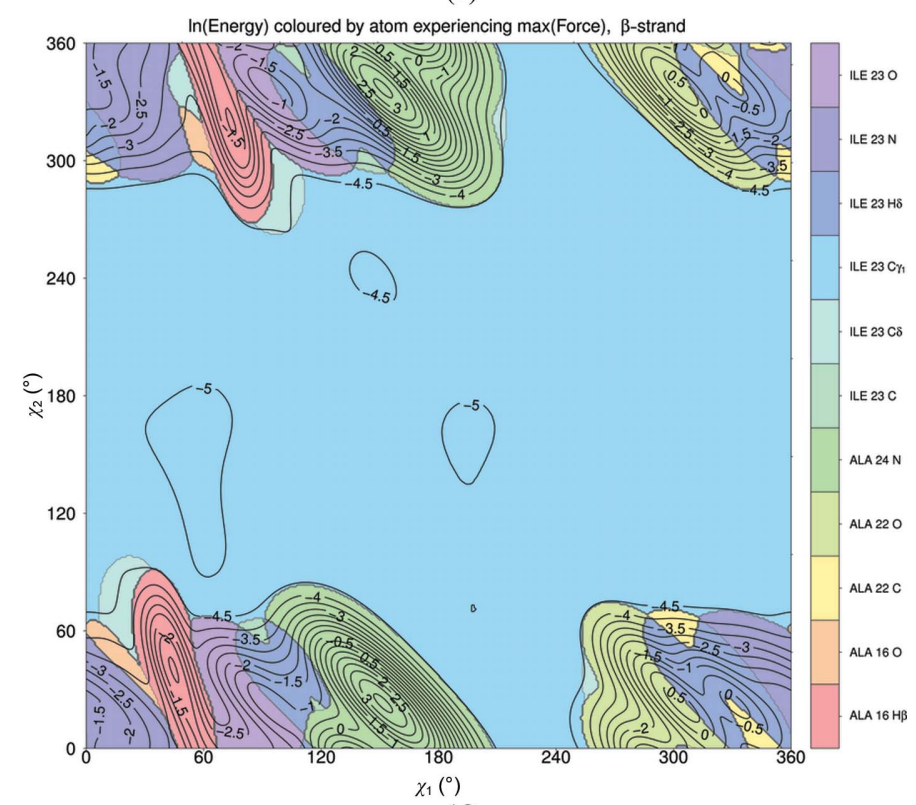

(d)

$(a, b)$ Energy-optimized starting situation of isoleucine modelled in a polyalanine helix $(a)\left(\chi_{1}, \chi_{2}=293,162^{\circ}\right)$ and an antiparallel polyalanine strand $(b)$ $\left(\chi_{1}, \chi_{2}=306,170^{\circ}\right) .(c)$ and $(d)$ show in colour for $360^{2}$ conformations which atom experiences the largest force using the YASARA NOVA force field for $\alpha$-helix and $\beta$-strand, respectively. Grey lines separate coloured areas. Black lines connect conformations with equal energy. $(e)$ and $(f)$ show for each conformation which pair of atoms shows the largest interatomic penetration for $\alpha$-helix and $\beta$-strand, respectively [1-3 interactions such as $\mathrm{C}^{\alpha}-\mathrm{C}^{\gamma}$ or $\mathrm{N}-\mathrm{C}^{\beta}$ are not included in $(e)$ and $(f)$ because they are invariable; side-chain protons are not used for clarity]. Note that different colouring schemes are used in $(c),(d),(e)$ and $(f) .(g)$ and $(h)$ show the frequency distribution of isoleucines in the nine sections in structures solved at better than $2.0 \AA$ resolution for $\alpha$-helix and $\beta$-strand, respectively. $(g)$ and $(h)$ are otherwise similar to Fig. 1. 
quantitative value because the YASARA NOVA force field was not used in the $\mathrm{X}$-ray refinement protocols. However, when we produce plots such as that shown in Fig. 1 for X-ray structures solved at better than $2.0 \AA$ resolution and exclusively refined with either CNS (Brünger et al., 1998) or REFMAC (Murshudov et al., 2011), we find that the local optima observed in the $\beta$-strand are about $1^{\circ}$ further away from YASARA's unfavourable areas in the $C N S$ structures than in the REFMAC structures. This is evident, for example, in sections 3 and 9 , which contain the most observed isoleucine $\chi_{1}-\chi_{2}$ combinations in areas that YASARA considers unfavourable.

Fig. 9 shows the behaviour of $\chi_{1}$ and $\chi_{2}$ as a function of the resolution and the refinement software used for isoleucines in

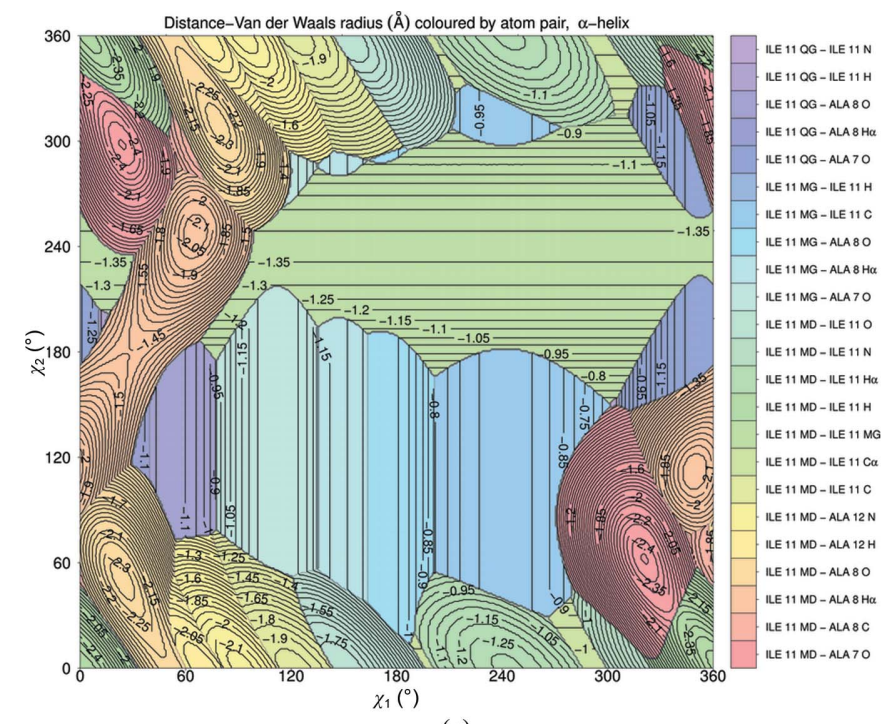

(e)

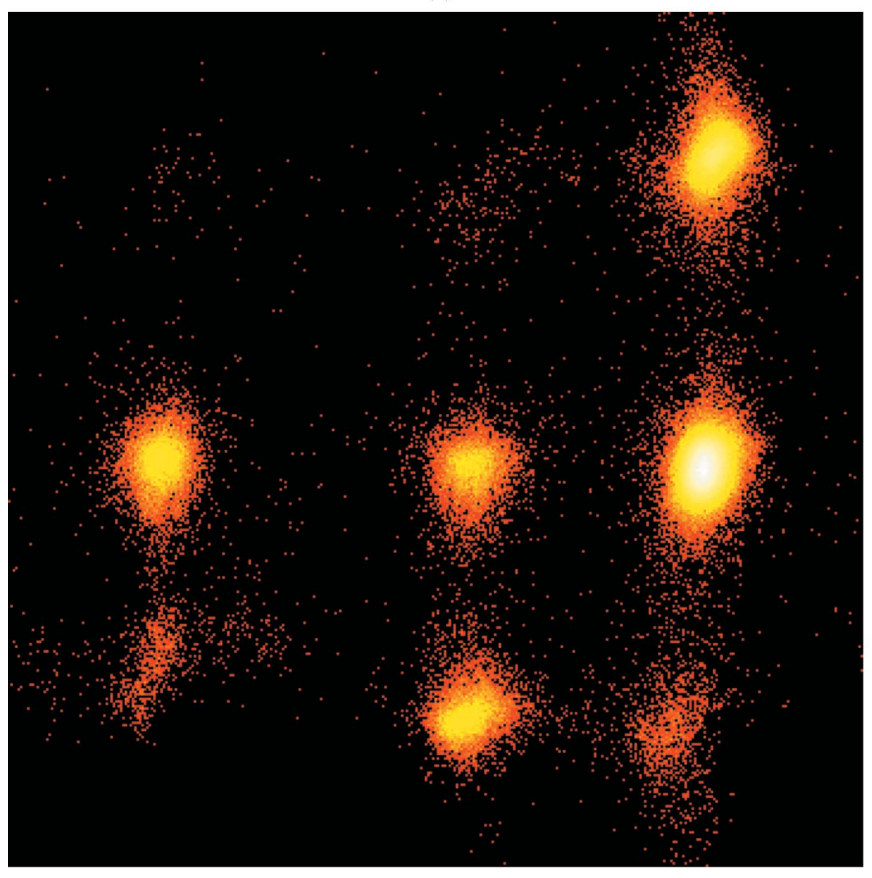

(g) an $\alpha$-helix in section $6\left(\chi_{1}, \chi_{2} \simeq 300,180^{\circ}\right)$. While $\chi_{1}$ shows hardly any significant differences, the $\chi_{2}$ values for REFMAC and SHELXL (Sheldrick, 2008) show systematic deviations from the values observed for the programs that use molecular dynamics-based force fields [X-PLOR (Brünger, 1992), CNS (Brünger et al., 1998)] or other forms of torsion-angle restraint (PHENIX; Adams et al., 2010).

\section{Discussion}

Despite rapidly approaching the milestone of 100000 entries, the PDB still does not contain sufficient data to allow us to make the detailed subset selections needed to answer some of the remaining questions. In future studies, we want to use

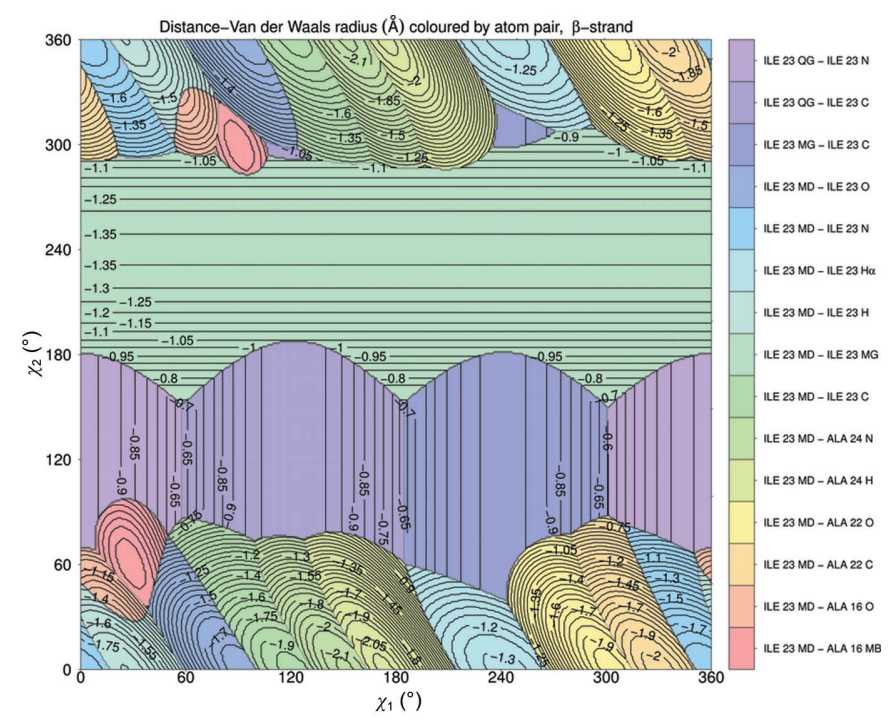

$(f)$

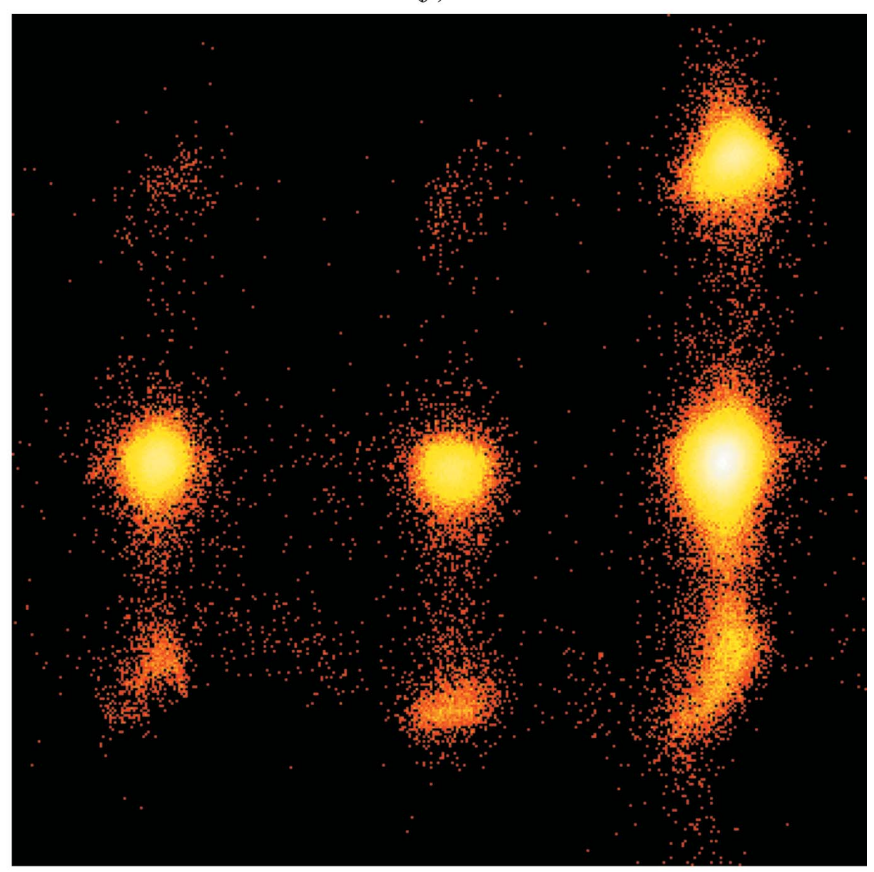

(h)

Figure 7 (continued) 


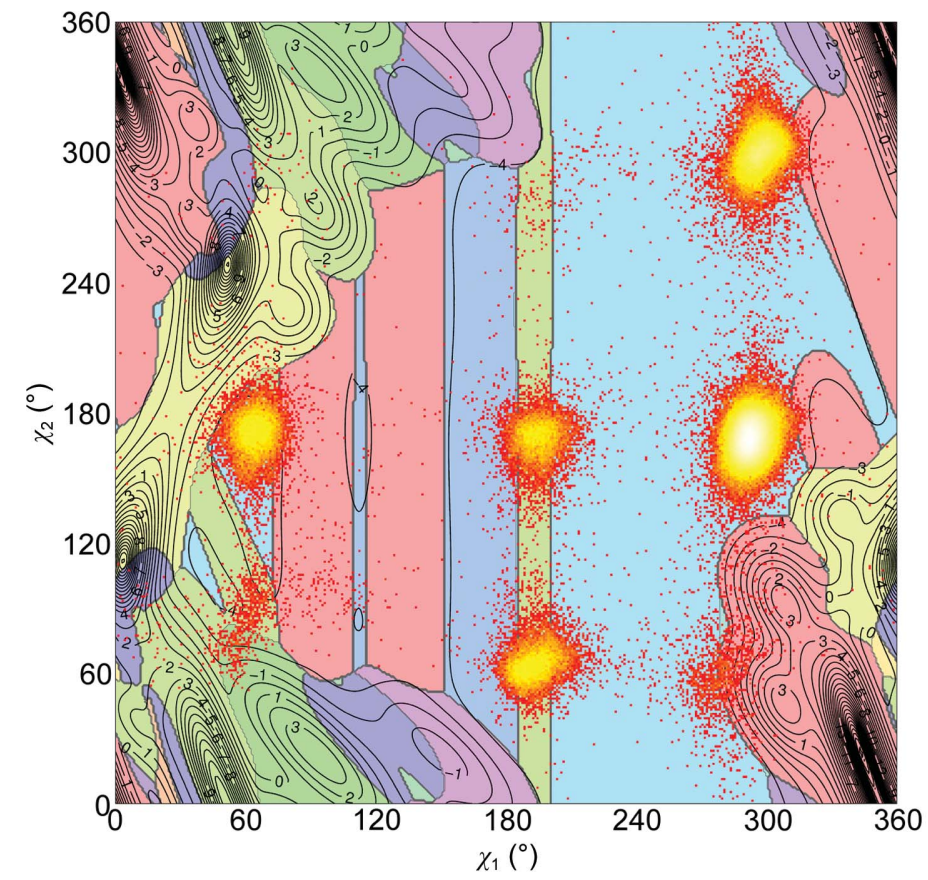

(a)

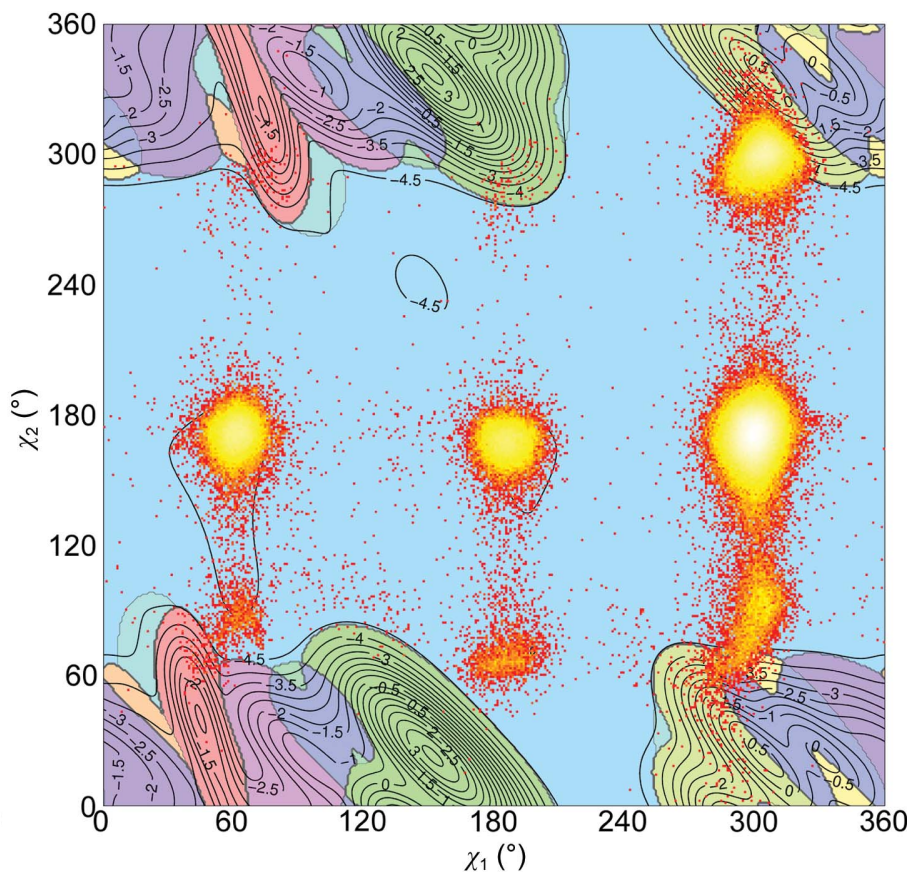

(b)

Figure 8

Isoleucines in an $\alpha$-helix (left) or in a $\beta$-strand (right). This figure was generated by superimposing Figs. $7(g)$ and $7(h)$ on Figs. $7(c)$ and $7(d)$, respectively.
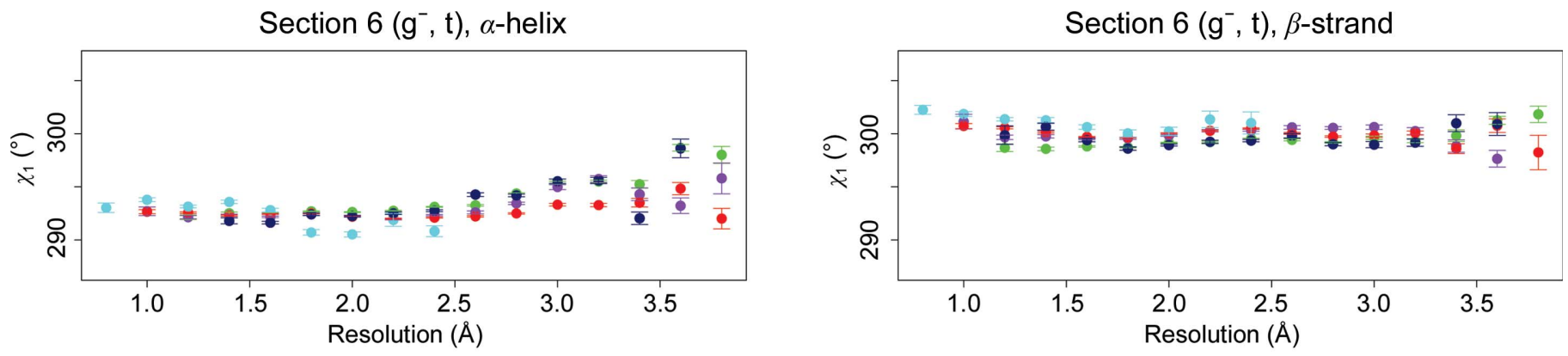

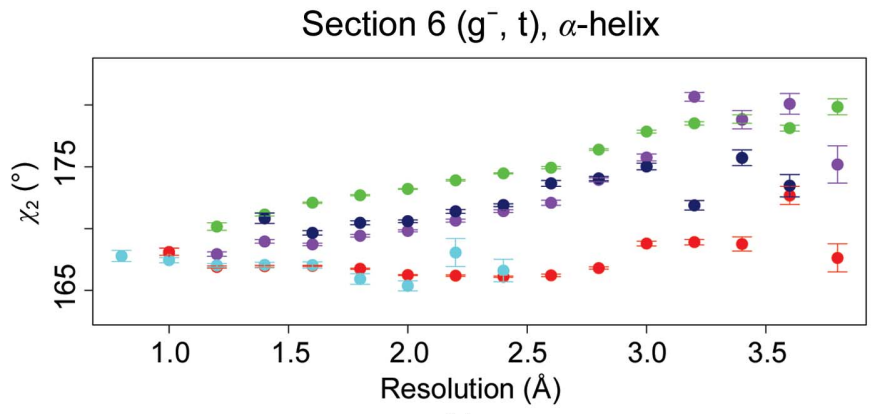

(a)

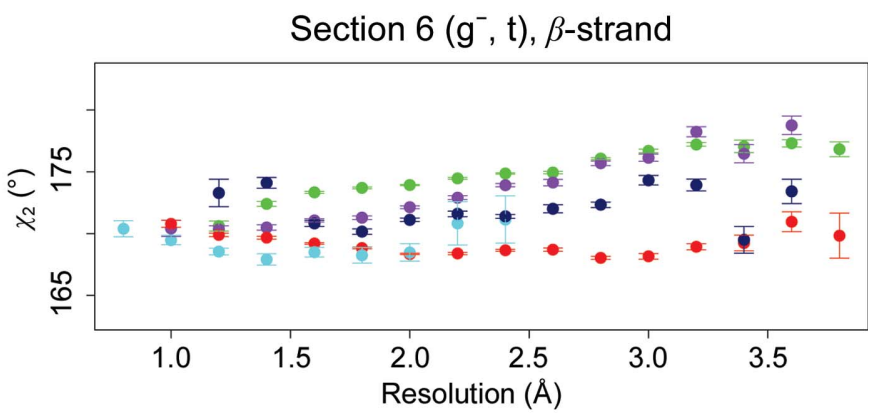

(b)

Figure 9

$\chi_{1}$ (top) and $\chi_{2}$ (bottom) as a function of the resolution and refinement software employed for isoleucines in an $\alpha$-helix (left) and in a $\beta$-strand (right). These $\chi_{1}$ and $\chi_{2}$ values correspond to section 6, which is the most populated section in all cases (more than half of all isoleucines fall in this section). REFMAC (Murshudov et al., 2011; red) and SHELXL (Sheldrick, 2008; light blue) behave similarly and so do the packages that explicitly use molecular dynamics-based force fields (X-PLOR, Brünger, 1992, dark blue; CNS, Brünger et al., 1998, green). PHENIX (Adams et al., 2010) results are in purple. Error bars represent the standard errors of the mean.

culled data sets that contain only one copy of a particular series of similar structures that were solved by the same group using the same software. This approach was taken when selecting the data underlying Fig. 4, but the associated reduction in available data (a factor of 5-10) was unacceptably large. In many of the panels in Figs. 2, 4, 5, 6 and 9, dots are missing because we (arbitrarily) require each dot to represent at least 50 observations. A reduction in the number of PDB files by a factor of 5-10 would greatly increase the number of missing observations unless we correspondingly reduced the number of data points per dot (and thus increased the noise) in these figures. 
It is practically impossible to determine how refinement programs and their sets of target values and/or force fields have changed over the years. When we split the data into two sets (structures solved before 2005 versus structures solved after 2004), we observe much smaller differences between the years than between secondary-structure elements. These data are available from the associated website (http:// swift.cmbi.ru.nl/gv/isoleucine/).

The SCRWL rotamer library described by Dunbrack \& Karplus (1993) uses the backbone torsion angles $\varphi$ and $\psi$ as a basis for rotamer prediction. Touw \& Vriend (2010) showed that the cooperative effect of secondary-structure elements causes $\tau$ angles to be systematically different between residues in $\alpha$-helix or $\beta$-strand and residues in a loop that have the same backbone torsion angles as residues in an $\alpha$-helix or $\beta$-strand. Figs. 5 and 6 show that a similar effect is observed for the relationship between side-chain rotamer preference and backbone torsion angles. The larger number of interactions involved in the side-chain rotamer choices, illustrated in Figs. 7 and 8 , makes this effect less straightforward to explain for the isoleucine $\chi_{1}, \chi_{2}$ angles than it was for $\tau$ angles.

The YASARA NOVA (Krieger et al., 2002) force-field set is based on the original Amber (Cornell et al., 1995) force-field set. Like other force-field sets such as CHARMM (MacKerell et al., 1998) or GROMACS (Hess et al., 2008), the Amber set is not complete. For example, it does not include terms such as induced polarization or higher order moments of aromatic planes. YASARA's force-field parameters have been extensively optimized to cope with these problems by minimizing the r.m.s. difference between high-resolution X-ray structures and homology models before and after short MD simulations. Consequently, the YASARA force field relies less on the preconceptions of basic physics and keeps proteins closer to reality than the original Amber force field. CNS (Brünger et al., 1998) and X-PLOR (Brünger, 1992) use energy force-field parameters that are somewhat similar to the values used by YASARA, while PHENIX (Adams, 2010) can use periodic torsion-angle restraints that perform a similar function. The resulting forces push the atoms in isoleucine side chains in several sections of the $\chi_{1}, \chi_{2}$ plot slightly away from their real position. At a resolution of around $1.0 \AA$ this effect is negligible, because the X-ray data will decide where the atoms end up. However, at a resolution of $2 \AA$ this effect is already significant, and at lower resolutions we observe differences of up to $10^{\circ}$. Side-chain torsion angle rotations of a few degrees in short side chains such as isoleucine will not lead to biologically significant errors in atomic positions. The errors enforced on the coordinates by the force field employed are nevertheless systematic in nature. It seems that refinement programs that use molecular dynamics-like energy parameters can be improved by adding more realistic, structure analysisbased, force-field terms similar to those incorporated into YASARA for the purpose of optimizing homology models (Krieger et al., 2002).

To assist the developers of refinement software, we have determined the $\chi_{1}, \chi_{2}$ optima and their two-dimensional spreads for each section and each secondary-structure type in high-resolution structures. The loop residues have been split into three categories: loops with helical $\varphi, \psi$ angles, loops with strand $\varphi, \psi$ angles and loops with other $\varphi, \psi$ angles. The resulting values show that it is better to use secondarystructure elements when a $\alpha$-helix or $\beta$-strand is observed and $\varphi, \psi$ angles in loops. A revised approach to torsion-angle restraints, possibly including correlated torsion-angle restraints, might need to be introduced in refinement software packages to optimally use these database-derived potentials.

We thank M. Hekkelman, B. van Kampen, T. te Beek and W. Touw for support. We thank Robbie Joosten for critically reading this manuscript and for incorporating the results in the REFMAC software. Erik Stens designed the relational database for protein structures. We thank the three anonymous referees for a large number of good suggestions. This work received financial support from the NewProt project, which is funded by the European Commission within its FP7 Programme, under the thematic area KBBE-2011-5 with contract No. 289350.

\section{References}

Adams, P. D. et al. (2010). Acta Cryst. D66, 213-221.

Berjanskii, M. V., Neal, S. \& Wishart, D. S. (2006). Nucleic Acids Res. 34, W63-W69.

Brünger, A. T. (1992). X-PLOR v.3.1. A System for X-ray Crystallography and NMR. Yale University, Connecticut, USA.

Brünger, A. T., Adams, P. D., Clore, G. M., DeLano, W. L., Gros, P., Grosse-Kunstleve, R. W., Jiang, J.-S., Kuszewski, J., Nilges, M., Pannu, N. S., Read, R. J., Rice, L. M., Simonson, T. \& Warren, G. L. (1998). Acta Cryst. D54, 905-921.

Brzozowski, A. M., Savage, H., Verma, C. S., Turkenburg, J. P., Lawson, D. M., Svendsen, A. \& Patkar, S. (2000). Biochemistry, 39, 15071-15082.

Butterfoss, G. L., Richardson, J. S. \& Hermans, J. (2005). Acta Cryst. D61, 88-98.

Chakrabarti, P. \& Pal, D. (2001). Prog. Biophys. Mol. Biol. 76, 1-102. Chen, V. B., Arendall, W. B., Headd, J. J., Keedy, D. A., Immormino, R. M., Kapral, G. J., Murray, L. W., Richardson, J. S. \& Richardson, D. C. (2010). Acta Cryst. D66, 12-21.

Clore, G. M. \& Kuszewski, J. (2002). J. Am. Chem. Soc. 124, 28662867.

Cornell, W. D., Cieplak, P., Bayly, C. I., Gould, I. R., Merz, K. M. Jr, Ferguson, D. M., Spellmeyer, D. C., Fox, T., Caldwell, J. W. \& Kollman, P. A. (1995). J. Am. Chem. Soc. 117, 5179-5197.

Dunbrack, R. L. Jr \& Cohen, F. E. (1997). Protein Sci. 6, 1661-1681.

Dunbrack, R. L. Jr \& Karplus, M. (1993). J. Mol. Biol. 230, 543-574.

Emsley, P. \& Cowtan, K. (2004). Acta Cryst. D60, 2126-2132.

Engh, R. A. \& Huber, R. (1991). Acta Cryst. A47, 392-400.

Hekkelman, M. L., Te Beek, T. A. H., Pettifer, S. R., Thorne, D., Attwood, T. K. \& Vriend, G. (2010). Nucleic Acids Res. 38, W719W723.

Hess, B., Kutzner, C., van der Spoel, D. \& Lindahl, E. (2008). J. Chem. Theory Comput. 4, 435-447.

Hooft, R. W. W., Sander, C. \& Vriend, G. (1996a). Comput. Appl. Biosci. 12, 525-529.

Hooft, R. W. W., Sander, C. \& Vriend, G. (1996b). J. Appl. Cryst. 29, 714-716.

Hooft, R. W. W., Sander, C. \& Vriend, G. (1996c). Proteins, 26, 363-376.

Hooft, R. W. W., Sander, C. \& Vriend, G. (1997). Comput. Appl. Biosci. 13, 425-430.

Hooft, R. W. W., Vriend, G., Sander, C. \& Abola, E. E. (1996). Nature (London), 381, 272. 
Jaskolski, M., Gilski, M., Dauter, Z. \& Wlodawer, A. (2007). Acta Cryst. D63, 611-620.

Jiang, F., Han, W. \& Wu, Y. (2010). J. Phys. Chem. B, 114, 5840-5850.

Jones, T. A. \& Thirup, S. (1986). EMBO J. 5, 819-822.

Joosten, R. P., Joosten, K., Cohen, S. X., Vriend, G. \& Perrakis, A. (2011). Bioinformatics, 27, 3392-3398.

Joosten, R. P., te Beek, T. A. H., Krieger, E., Hekkelman, M. L., Hooft, R. W. W., Schneider, R., Sander, C. \& Vriend, G. (2011). Nucleic Acids Res. 39, D411-D419.

Joosten, R. P. \& Vriend, G. (2007). Science, 317, 195-196.

Kabsch, W. \& Sander, C. (1983). Biopolymers, 22, 2577-2637.

Karplus, P. A. (1996). Protein Sci. 5, 1406-1420.

Kimball, S., Mattis, P., Natterer, M. \& Neumann, S. (1997). GIMP: GNU Image Manipulation Program, v.2.6.8. http://www.gimp.org/.

Kleywegt, G. J., Harris, M. R., Zou, J., Taylor, T. C., Wählby, A. \& Jones, T. A. (2004). Acta Cryst. D60, 2240-2249.

Krieger, E., Dunbrack, R. L. Jr, Hooft, R. W. \& Krieger, B. (2012). Methods Mol. Biol. 819, 405-421.

Krieger, E., Koraimann, G. \& Vriend, G. (2002). Proteins, 47, 393-402.

Laskowski, R. A., MacArthur, M. W., Moss, D. S. \& Thornton, J. M. (1993). J. Appl. Cryst. 26, 283-291.

Laskowski, R. A., Moss, D. S. \& Thornton, J. M. (1993). J. Mol. Biol. 231, 1049-1067.

Lindorff-Larsen, K., Piana, S., Palmo, K., Maragakis, P., Klepeis, J. L., Dror, R. O. \& Shaw, D. E. (2010). Proteins, 78, 1950-1958.

Lovell, S. C., Davis, I. W., Arendall, W. B., de Bakker, P. I. W., Word, J. M., Prisant, M. G., Richardson, J. S. \& Richardson, D. C. (2003). Proteins, 50, 437-450.

Lovell, S. C., Word, J. M., Richardson, J. S. \& Richardson, D. C. (2000). Proteins, 40, 389-408.

Lundgren, M. \& Niemi, A. J. (2012). Phys. Rev. E, 86, 021904.

Lutz, M. (2001). Programming Python, 2nd ed. Sebastopol: O'Reilly.
MacArthur, M. W. \& Thornton, J. M. (1996). J. Mol. Biol. 264, 11801195.

MacArthur, M. W. \& Thornton, J. M. (1999). Acta Cryst. D55, 994 1004.

MacKerell, A. D. et al. (1998). J. Phys. Chem. B, 102, 35863616.

Murshudov, G. N., Skubák, P., Lebedev, A. A., Pannu, N. S., Steiner, R. A., Nicholls, R. A., Winn, M. D., Long, F. \& Vagin, A. A. (2011). Acta Cryst. D67, 355-367.

Ponder, J. W. \& Richards, F. M. (1987). J. Mol. Biol. 193, 775-791.

R Development Core Team (2012). R: A Language and Environment for Statistical Computing. R Foundation for Statistical Computing, Vienna, Austria. http://www.R-project.org/.

Read, R. J. et al. (2011). Structure, 19, 1395-1412.

Reiling, K. K., Endres, N. F., Dauber, D. S., Craik, C. S. \& Stroud, R. M. (2002). Biochemistry, 41, 4582-4594.

Rice, L. M. \& Brünger, A. T. (1994). Proteins, 19, 277-290.

Rosenwirth, B., Oren, D. A., Arnold, E., Kis, Z. L. \& Eggers, H. J. (1995). Antiviral Res. 26, 65-82.

Schmidt, A., Teeter, M., Weckert, E. \& Lamzin, V. S. (2011). Acta Cryst. F67, 424-428.

Sheldrick, G. M. (2008). Acta Cryst. A64, 112-122.

Sychrovsky, V., Foldynova-Trantirkova, S., Spackova, N., Robeyns, K., Van Meervelt, L., Blankenfeldt, W., Vokacova, Z., Sponer, J. \& Trantirek, L. (2009). Nucleic Acids Res. 37, 7321-7331.

Touw, W. G. \& Vriend, G. (2010). Acta Cryst. D66, 1341-1350.

Tsai, C. S. (2007). Biomacromolecules: Introduction to Structure, Function and Informatics, p. 4. Hoboken: John Wiley \& Sons.

Vriend, G. (1990). J. Mol. Graph. 8, 52-56.

Wang, Q., Canutescu, A. A. \& Dunbrack, R. L. Jr (2008). Nature Protoc. 3, 1832-1847.

Wickham, H. (2011). J. Stat. Softw. 40. http://www.jstatsoft.org/v40/ i01. 